\title{
Effect of Revised Nursery Orders on Newborn Preventive Services
}

\author{
Diane J. Madlon-Kay, MD, MS
}

Objective: Aspects of neonatal care that are the subject of evolving guidelines include hepatitis B virus (HBV) immunization; discharge follow-up recommendations; and prevention of perinatal group B streptococcal (GBS) disease. In 2007, a university hospital's standardized newborn nursery orders were changed to reflect current recommendations in these areas. The objective of the study was to determine the effect of new nursery orders on the quality of care provided to these newborns.

Methods: The study was a retrospective review of medical records, birth certificates, and a computer database of 857 infants. The nursery orders changed in the following ways: (1) physicians had to "opt out" of HBV immunization; (2) discharge follow-up recommendations were based on American Academy of Pediatrics (AAP) recommendations; and (3) AAP recommendations for GBS were followed except blood cultures were not required for certain infants.

Results: The percentage of infants receiving $\mathrm{HBV}$ immunizations increased from $74 \%$ in 2007 to $83 \%$ in $2008(P=.0018)$. The percentage of infants whose mothers received antibiotics for GBS less than 4 hours before delivery and who received a complete blood count increased from $36 \%$ to $83 \%(P<$ .0001). The percentage of newborns who had discharge follow-up plans consistent with AAP recommendations did not change significantly.

Conclusion: A simple change in nursery orders was associated with significant improvement in newborn care. (J Am Board Fam Med 2011;24:656-664.)

Keywords: Infant, Newborn Care, Practice Guidelines, Preventive Health Services, Quality Improvement

Guidelines are often used to improve care for patients of all ages. The American Academy of Pediatrics (AAP) published new recommendations in 2010 regarding the services that should be provided to newborns before hospital discharge. ${ }^{1}$ These services include immunization for hepatitis $\mathrm{B}$ virus (HBV) and arrangements for appropriate followup. The AAP also has endorsed other guidelines that affect the care of newborns in the hospital, including one that addresses the prevention of perinatal group B streptococcal (GBS) disease. ${ }^{2}$

This article was externally peer reviewed.

Submitted 27 January 2011; revised 18 April 2011; accepted 25 April 2011.

From the Department of Family Medicine and Community Health, University of Minnesota Medical School, Minneapolis, MN.

Funding: Support has been provided by the UCare Fund of the Minnesota Medical Foundation.

Conflict of interest: none declared.

Corresponding author: Diane J. Madlon-Kay, 2020 East 28th Street, Minneapolis, MN 55407 (E-mail: madlo001@tc.umn.edu).
There seems to be only one previous publication of an effort to improve multiple newborn preventive services during the birth hospitalization. In Vermont, all hospitals with obstetric services participated in a statewide, collaborative quality improvement project targeting 13 preventive services delivered to healthy newborns during the birth hospitalization. ${ }^{3}$ At the end of an 18-month period, seven services improved with the intervention. No significant changes were noted for the administration of HBV immunization or for the documentation of the date of planned newborn follow-up.

This intervention was quite labor intensive and expensive. Therefore, it is questionable whether it will be adopted or sustained in many newborn nursery settings. The purpose of this study was to determine the effect of a potentially simple and efficient method of improving quality to newborns: changing routine nursery orders.

In October 2007, the standardized orders at a university hospital nursery were changed to better reflect current care guidelines in the following 
three areas: (1) HBV immunization; (2) discharge follow-up recommendations, and (3) management of newborns exposed to GBS prophylaxis. These changes are summarized in Table 1 . The hospital newborn nursery committee decided not to include a standard order for a blood culture for infants whose mothers received intrapartum antibiotic prophylaxis (IAP) for GBS less than 4 hours before delivery. This decision was made after reviewing how other nurseries were responding to this recommendation and reviewing the barriers to its implementation.

The specific measures evaluated were (1) the percentage of infants who received $\mathrm{HBV}$ vaccine while in the nursery; (2) the percentage of newborns who had discharge follow-up plans consistent with the AAP recommendations ${ }^{4}$; and (3) the percentage of infants exposed to IAP for GBS who received the evaluation and length of stay (LOS) recommended by the Centers for Disease Control and Prevention (CDC). ${ }^{2}$

\section{Methods}

The study was a retrospective review of medical records, birth certificate data, and a computer database for 857 newborns at Fairview Riverside Hospital in Minneapolis, Minnesota. In 2007, 2339 infants were cared for in the hospital's normal newborn nursery. An additional 537 infants were in neonatal intensive care. The hospital does not have a level II nursery. Newborn care is provided by community pediatricians and family physicians, as well as residents and faculty from a family medicine residency. The hospital provides care for a racially and socioeconomically diverse population, including many Somali refugees.

Nursery order sets are used for all newborns in the normal newborn nursery. The order sets were printed on paper until May 20, 2008. After that date, the order sets were electronic. A paper chart continued to be used for all other aspects of the infant's care. When a baby is born, the nursery nurse initiates care, using the admission order set. The physician signs the admission order set when he or she first examines the infant. The physician completes and signs a discharge order set when he or she discharges the infant.

Newborns born at Fairview Riverside Hospital from January through June 2007 and from January through June 2008 were eligible for inclusion in the study. Because the new nursery orders were implemented in October 2007, nursery staff and physicians had several months to adjust to the change. For the last 6 weeks of the study, the order sets were electronic rather than paper.

A simple random sample of 857 infants-434 born before and 423 born after the change in nursery orders-were identified from the Patient Administrative Services and Systems (PASS) computer database. Infants born at less than 35 weeks' estimated gestational age (EGA) were excluded because they do not receive care in the normal newborn nursery. CDC guidelines recommend that infants born to mothers receiving antibiotics for suspected chorioamnionitis receive empiric antibiotic therapy. At the time of the study, empiric

Table 1. Overview of Newborn Nursery Standardized Order Changes

\begin{tabular}{|c|c|c|}
\hline Outcome & Old Nursery Orders & New Nursery Orders \\
\hline $\begin{array}{l}\text { Hepatitis B virus } \\
\text { vaccination }\end{array}$ & $\begin{array}{l}\text { Physicians must "opt in," ie, specifically } \\
\text { order the vaccine }\end{array}$ & $\begin{array}{l}\text { Physicians must "opt out," ie, specifically } \\
\text { order that no vaccine be given }\end{array}$ \\
\hline $\begin{array}{l}\text { Discharge follow-up } \\
\text { recommendations }\end{array}$ & No guidance & $\begin{array}{l}\text { Recommendations based on infant's age } \\
\text { at discharge, transcutaneous bilirubin } \\
\text { result before discharge, and jaundice risk } \\
\text { factors, including breastfeeding. Follow- } \\
\text { up intervals based on AAP } \\
\text { recommendations. }\end{array}$ \\
\hline GBS disease prevention & $\begin{array}{l}\text { If the mother was positive for GBS, } \\
\text { infant's vital signs were measured more } \\
\text { frequently }\end{array}$ & $\begin{array}{l}\text { Follow CDC recommendations including } \\
\text { observation for } 48 \text { hours for many } \\
\text { infants. }^{2} \\
\text { Orders differ from CDC guideline by } \\
\text { requiring only complete blood counts, } \\
\text { and not blood cultures, for certain } \\
\text { infants. }\end{array}$ \\
\hline
\end{tabular}

GBS, group B streptococcal; AAP, American Academy of Pediatrics; CDC, Centers for Disease Control and Prevention. 
antibiotic therapy was given only in neonatal intensive care because the hospital does not have a level II nursery. Therefore, infants born at $\geq 35$ weeks EGA being cared for in neonatal intensive care were included for the evaluation of GBS disease prevention.

The principal investigator (DMK) trained the chart reviewer. For quality assurance, a random sample of $10 \%$ of charts was examined by both the reviewer and the principal investigator. The study was approved by the University of Minnesota Institutional Review Board.

\section{Outcome Measures}

\section{HBV Immunization}

The percentage of infants receiving $\mathrm{HBV}$ immunizations was determined by chart review.

\section{Discharge Follow-up Recommendations}

Date and time of birth was obtained from birth certificate data. Discharge date and time was obtained from the PASS database. Documentation of discharge follow-up was determined by chart review of the discharge orders, ie, which discharge follow-up option was chosen by the physician. We determined the percentage of infants who were scheduled appropriately for follow-up according to the AAP recommendations. The AAP recommends that infants discharged before 24 hours be seen by age 72 hours. ${ }^{4}$ Infants with an LOS of between 24 and 47.9 hours should be seen by age 96 hours, and infants discharged between 48 and 72 hours should be seen by age 120 hours.

\section{GBS Disease Prevention}

The new nursery orders contained the following regarding GBS:

- Providers caring for infants whose mothers received antibiotics for suspected chorioamnionitis were to be notified to consider neonatal intensive care evaluation and transfer for initiation of intravenous antibiotics.

- Infants whose mothers received IAP for GBS $<4$ hours before delivery received a complete blood count (CBC) with differential and were to be observed for at least 48 hours before discharge.

- Infants whose mothers received IAP for GBS $\geq 4$ hours before delivery were to be observed for at least 48 before discharge.
Infants' charts were reviewed to determine whether the mother received antibiotics during labor and, if so, the name of the antibiotic and time between the first dose and delivery. If the mother received a typical antibiotic used for GBS prophylaxis (penicillin, ampicillin, cefazolin, clindamycin, erythromycin, or vancomycin), the infant was classified as having maternal IAP for GBS. ${ }^{2}$ If the mother had a documented fever of $100.4^{\circ} \mathrm{C}$ or higher and different antibiotics were used in labor, she was classified as having received antibiotics for suspected chorioamnionitis. Based on these classifications, GBS disease prevention measures were assessed as follows:

- For infants whose mothers received antibiotics for suspected chorioamnionitis, we determined the percentage who received full diagnostic evaluation, which included CBC with differential, blood culture, possible chest radiograph, possible lumbar puncture, and empiric antibiotic therapy.

- For infants who appeared healthy but whose mothers received IAP for GBS $<4$ hours before delivery, we determined the percentage who received a CBC with differential and blood culture and who were observed for at least 48 hours.

- For infants who appeared healthy but whose mothers received IAP for GBS $\geq 4$ hours before delivery, we determined the percentage who were observed for 48 or more hours.

Information about the following variables was obtained from birth certificate data: mother and father's race/ethnicity, mother and father's country of birth, mother's education level, number of prenatal visits, infant birth weight, method of delivery, singleton versus twin, mother's age, infant's sex, maternal diabetes, and EGA. The mother's insurance and method of feeding were determined by chart review. The infant's LOS ( $<24$ hours, $24-47$ hours, 48-72 hours, and $>72$ hours) was determined from the PASS database.

\section{Statistical Analysis}

A sample size calculation determined that 413 infants per group would have greater than $90 \%$ power to detect differences in the three outcomes using Fisher's exact tests, with a two-sided significance level of $P=.05$. Infant and parent demographics and characteristics were summarized with descriptive statistics (means, SD, and range for continuous measures and counts and percents for cate- 
gorical measures). HBV immunization rates for the 2007 and 2008 newborn groups were compared using a $\chi^{2}$ test. In addition, a logistic regression model was used to assess the impact of race/ethnicity on HBV immunizations. For the discharge recommendations outcome, follow-up was summarized and rates were compared between groups using a Fisher's exact test. Discharge follow-up was also summarized by LOS. Mean time until follow-up was compared between groups using a Student's $t$ test. Evaluation and LOS for GBS disease prevention were compared between groups using Fisher's exact tests. Two-sided $P<.05$ were deemed statistically significant. SAS software version 9.1.3 (SAS Institute, Cary, NC) was used for the analyses.

\section{Results}

Demographic information about the infants and parents is shown in Table 2. A large number of immigrant women give birth at the hospital, especially refugees from Somalia. These women typically have little formal education. Eighty-six infants received care in the neonatal intensive care unit. The only outcome measure evaluated among these infants was GBS prevention. Seven hundred seventyone infants received care in the normal newborn nursery, so all three outcomes were studied in these newborns.

\section{HBV Immunization}

HBV immunization status was available for the 771 infants who received care in the normal newborn nursery. The percentage of infants receiving HBV immunizations increased from $73 \%$ in 2007 to $84 \%$ in $2008(P=.0004)$. Overall, white infants had a lower HBV immunization rate $(68 \%)$ than nonwhite infants $(84 \%)(P<.0001)$. White infants were defined as having two white parents.

\section{Discharge Follow-up Recommendations}

In 2007, although no guidance was provided regarding the appropriate time for discharge follow-up in the clinic, the standardized discharge order form did provide options from which the physician could choose: 2 to 5 days or 7 to 14 days. The order form also included a place where the physician could indicate a different number of days until clinic follow-up.

In 2008, the options provided on the standardized discharge order form were 24 hours, 48 hours, and 2 to 3 days. Each of these options were accompanied by a description of which infants should have each follow-up interval based on AAP recommendations. ${ }^{4}$ The physicians still had an "other" option to indicate a different number of days until follow-up.

Table 3 shows the clinic follow-up recommendations in 2007 and 2008. There was no significant change in appropriate discharge follow-up. A persistent minority of infants had clinic follow-up recommended a week or more after discharge. The mean time until follow-up was determined for babies discharged before 72 hours. When a range of follow-up time was recommended, the earliest follow-up time was included in this analysis. The mean \pm SD time was not significantly improved $(P=.27)$, with $119.4 \pm 49.8$ hours until follow-up in 2007 and $115.5 \pm 48.2$ hours in 2008 .

\section{GBS Disease Prevention}

Mothers with a documented temperature $\geq 100.4^{\circ} \mathrm{C}$ and who received an antibiotic other than the six typically used for GBS prophylaxis were considered to have received antibiotics for suspected chorioamnionitis. There were seven such mothers in 2007 and 10 in 2008. Figure 1 demonstrates the diagnostic evaluation and therapy given to the infants born to these mothers. There was no significant change in the percentage of infants who received the care recommended by the CDC guideline. ${ }^{2}$

Figure 2 demonstrates the evaluation and LOS of infants in the normal newborn nursery whose mothers received IAP for GBS prevention. The 2008 standardized orders recommended CBC only for evaluation of infants whose mothers received IAP $<4$ hours before delivery, whereas the CDC guidelines also recommended blood cultures. In 2007,10 of 33 of these infants (30\%) received both a CBC and blood culture. In 2008, significantly more of these infants had a CBC and significantly fewer had a blood culture. There was no change in the percentage of these infants who had the 48hour LOS recommended by the CDC.

Similarly, there was no improvement in the percentage of babies with an LOS $\geq 48$ hours among those infants whose mothers received IAP for GBS prevention $\geq 4$ hours before delivery (61\% in 2007 vs $56 \%$ in $2008 ; P=.60$ ). There was no increase in LOS even if these infants had an EGA of 35 to 37 weeks $(50 \%$ in 2007 vs $67 \%$ in $2008 ; P=.65)$. 


\begin{tabular}{|c|c|c|c|}
\hline & Statistic & $2007(\mathrm{n}=434)$ & $2008(n=423)$ \\
\hline \multicolumn{4}{|l|}{ Infant Sex } \\
\hline Male & $\mathrm{n}(\%)$ & $221(51)$ & $208(49)$ \\
\hline \multirow[t]{2}{*}{ Birth weight (g) } & Mean (SD) & $3407(510.7)$ & $3416(505.6)$ \\
\hline & Range & $1814-4990$ & $1980-4884$ \\
\hline Singleton birth & $\mathrm{n}(\%)$ & $425(98)$ & $415(98)$ \\
\hline \multirow[t]{2}{*}{ APGAR score at 1 minute } & Mean (SD) & $8(1.4)$ & $8(1.4)$ \\
\hline & Range & 1 to 10 & 1 to 9 \\
\hline \multirow[t]{2}{*}{ APGAR score at 5 minutes } & Mean (SD) & $9(0.5)$ & $9(0.5)$ \\
\hline & Range & 5 to 10 & 4 to 10 \\
\hline \multicolumn{4}{|l|}{ Method of birth } \\
\hline Vaginal & $\mathrm{n}(\%)$ & $306(71)$ & $297(70)$ \\
\hline Assisted vaginal & $\mathrm{n}(\%)$ & $20(5)$ & $14(3)$ \\
\hline Caesarean section & $\mathrm{n}(\%)$ & $108(25)$ & $112(26)$ \\
\hline \multirow[t]{2}{*}{ Length of stay (hours) } & Median (SD) & $54(96.3)$ & $52(60.5)$ \\
\hline & Range & 13 to 1754 & 15 to 917 \\
\hline$<24$ & $\mathrm{n}(\%)$ & $1(0.2)$ & $3(1)$ \\
\hline $24-47$ & $\mathrm{n}(\%)$ & $152(35)$ & $158(37)$ \\
\hline $48-72$ & $\mathrm{n}(\%)$ & $160(37)$ & $149(35)$ \\
\hline$>72$ & $\mathrm{n}(\%)$ & $121(28)$ & $113(27)$ \\
\hline \multirow[t]{2}{*}{ Maternal age (years) } & Mean (SD) & $29(6.1)$ & $29(6.2)$ \\
\hline & Range & 15 to 50 & 15 to 46 \\
\hline \multicolumn{4}{|l|}{ Maternal education (years) } \\
\hline $0-12$ & $\mathrm{n}(\%)$ & $195(45)$ & $183(43)$ \\
\hline $13-16$ & $\mathrm{n}(\%)$ & $133(31)$ & $135(32)$ \\
\hline $17+$ & $\mathrm{n}(\%)$ & $106(24)$ & $105(25)$ \\
\hline \multicolumn{4}{|l|}{ Maternal race } \\
\hline White & $\mathrm{n}(\%)$ & $189(44)$ & $214(51)$ \\
\hline Black & $\mathrm{n}(\%)$ & $127(29)$ & $119(28)$ \\
\hline American Indian & $\mathrm{n}(\%)$ & $19(4)$ & $9(2)$ \\
\hline Asian & $\mathrm{n}(\%)$ & $42(10)$ & $47(11)$ \\
\hline Other & $\mathrm{n}(\%)$ & $75(17)$ & $47(11)$ \\
\hline Unknown & $\mathrm{n}(\%)$ & $2(0.5)$ & $4(1)$ \\
\hline \multicolumn{4}{|l|}{ Paternal race } \\
\hline White & $\mathrm{n}(\%)$ & $178(41)$ & $199(47)$ \\
\hline Black & $\mathrm{n}(\%)$ & $142(33)$ & $116(27)$ \\
\hline American Indian & $\mathrm{n}(\%)$ & $9(2)$ & $7(2)$ \\
\hline Asian & $\mathrm{n}(\%)$ & $38(9)$ & $42(10)$ \\
\hline Other & $\mathrm{n}(\%)$ & $72(17)$ & $50(12)$ \\
\hline Unknown & $\mathrm{n}(\%)$ & $12(3)$ & $24(6)$ \\
\hline \multicolumn{4}{|l|}{ Father Hispanic } \\
\hline Yes & $\mathrm{n}(\%)$ & $61(14)$ & $51(12)$ \\
\hline No & $\mathrm{n}(\%)$ & $349(80)$ & $347(82)$ \\
\hline Missing & $\mathrm{n}(\%)$ & $24(6)$ & $25(6)$ \\
\hline \multicolumn{4}{|l|}{ Mother Hispanic } \\
\hline Yes & $\mathrm{n}(\%)$ & $67(15)$ & $52(12)$ \\
\hline No & $\mathrm{n}(\%)$ & $354(82)$ & $366(87)$ \\
\hline Missing & $\mathrm{n}(\%)$ & $13(3)$ & $5(1)$ \\
\hline \multicolumn{4}{|l|}{ Father birthplace } \\
\hline United States & n (\%) & $229(53)$ & $240(57)$ \\
\hline \multirow[t]{2}{*}{ Somalia } & $\mathrm{n}(\%)$ & $56(13)$ & $46(11)$ \\
\hline & & & Continued \\
\hline
\end{tabular}


Table 2. Continued

\begin{tabular}{llcr}
\hline & Statistic & $2007(\mathrm{n}=434)$ & $2008(\mathrm{n}=423)$ \\
\hline Other & $\mathrm{n}(\%)$ & $149(34)$ & $137(32)$ \\
Mother birthplace & & & \\
United States & $\mathrm{n}(\%)$ & $251(58)$ & $267(63)$ \\
Somalia & $\mathrm{n}(\%)$ & $60(14)$ & $51(12)$ \\
Other & $\mathrm{n}(\%)$ & $123(28)$ & $105(25)$ \\
Prenatal visits & Mean (SD) & $10.3(3.2)$ & $10.7(3.0)$ \\
& Range & 1 to 24 & 0 to 21 \\
EGA (weeks) & Mean (SD) & $39.4(1.3)$ & $39.4(1.5)$ \\
& Range & 35.3 to 42.3 & 35 to 43.6 \\
Maternal diabetes & $\mathrm{n}(\%)$ & $40(9)$ & $34(8)$ \\
\hline
\end{tabular}

EGA, estimated gestational age.

\section{Discussion}

The change in the newborn nursery standardized orders was associated with improvement in delivery of some, but not all, newborn preventive services. The percentage of infants receiving hepatitis $\mathrm{B}$ vaccination increased significantly

Table 3. Discharge Follow-up Recommendations

\begin{tabular}{ccc}
\hline & 2007 & 2008 \\
\hline $\begin{array}{c}\text { LOS }<24 \text { hours } \\
\text { Clinic follow-up (days) }\end{array}$ & $\mathrm{n}=1$ & $\mathrm{n}=3$ \\
$1-5^{*}$ & $1(100)$ & $2(67)$ \\
$\geq 7^{\dagger}$ & 0 & 0 \\
LOS $24-47$ hours & $\mathrm{n}=152$ & $\mathrm{n}=155$ \\
Clinic follow-up (days) & & \\
1 to $5^{*}$ & $130(86)$ & $140(90)$ \\
$\geq 7^{\dagger}$ & $9(6)$ & $8(5)$ \\
LOS $48-72$ hours & $\mathrm{n}=151$ & $\mathrm{n}=138$ \\
Clinic follow-up (days) & $129(85)$ & $120(87)$ \\
$1-5^{*}$ & $10(7)$ & $9(7)$ \\
$\geq 7^{\dagger}$ & $\mathrm{n}=90$ & $\mathrm{n}=81$ \\
LOS $>72$ hours & & \\
Clinic follow-up (days) & $69(77)$ & $74(91)$ \\
$1-5^{*}$ & $10(11)$ & $3(4)$ \\
$\geq 7^{\dagger}$ & $\mathrm{n}=394$ & $\mathrm{n}=377$ \\
All & & $336(89)$ \\
Clinic follow-up & $329(84)$ & $20(5)$ \\
$1-5^{*}$ & $29(7)$ & \\
$\geq 7^{\dagger}$ & &
\end{tabular}

*Twenty-four hours, 48 hours, 2 to 3 days, or others that were $<4$ days for 2008; 2 to 5 days or others that were $<3$ days for 2007.

${ }^{\dagger}$ Others that were at least 7 days for $2008 ; 7$ to 14 days or others that were at least 7 days for 2007.

${ }^{\ddagger}$ Fisher's exact test; $P=.24$.

LOS, length of stay. from $73 \%$ to $84 \%(P=.0004)$. The percentage of infants whose mothers received IAP $<4$ hours before delivery who received a CBC increased significantly from $36 \%$ to $83 \%(P<.0001)$. However, there was no improvement in the percentage of infants who had discharge follow-up recommendations consistent with AAP recommendations.

The change in nursery orders was a relatively simple intervention. The hospital's normal newborn nursery committee recommended the changes, which were then disseminated via Email to all physicians providing nursery care for comment. At the time of implementation, nursery nurses and the hospital laboratory were educated about the changes. In contrast, the only other previously reported intervention to improve multiple preventive health care services for well newborns during the birth hospitalization was a statewide, collaborative, hospital-based quality-improvement project that took place over 18 months. ${ }^{3}$ Results were also mixed in that project, with improvements in 7 of 13 targeted preventive services.

Universal infant hepatitis B vaccination beginning at birth has been recommended since December 2005 to provide a "safety net" for the prevention of perinatal and early childhood infections. ${ }^{5}$ In a March 2006 survey of a nationally representative sample of delivery hospitals in the United States, $69.1 \%$ of infants born to hepatitis B surface antigen-negative mothers received the vaccine before hospital discharge. ${ }^{6}$ The percentage of infants who received the vaccine did not vary significantly by maternal race or ethnicity. The strongest predictor of vaccine administration was having a written hos- 
Figure 1. Change in compliance with American Academy of Pediatrics guidelines for mothers receiving antibiotics for suspected chorioamnionitis. CBC, complete blood count.

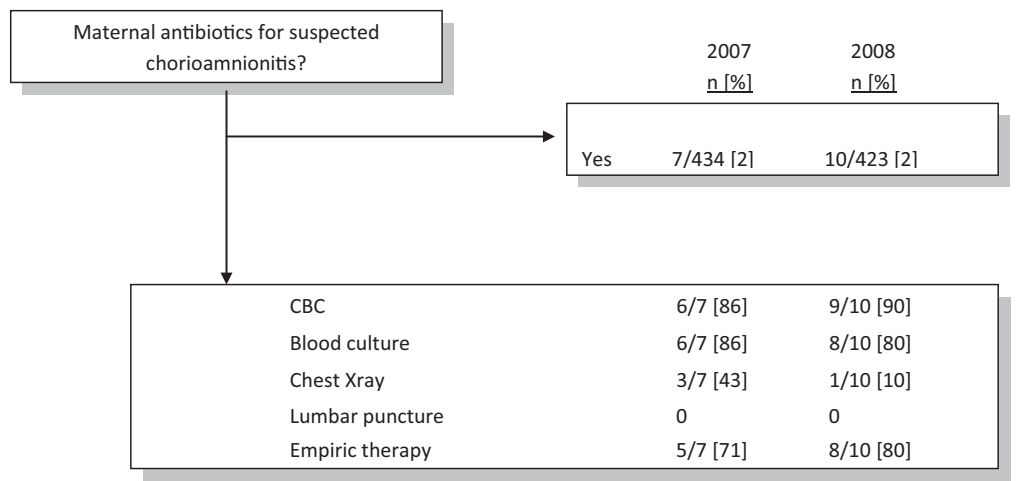

pital policy for universal HBV vaccine administration to newborns before hospital discharge. Sixtyseven percent of the 242 hospitals surveyed had such a policy.

Although the new nursery standardized orders included universal HBV vaccination, the physicians were still required to discuss the shot with the parents personally before the vaccine was given. Therefore, the $83 \%$ vaccination rate, though improved, was not as high as the $87.2 \%$ rate reported by hospitals with universal vaccination rates that were included in the survey. ${ }^{6}$ In addition, in our hospital, vaccination rates were lower for infants with two white parents. The newborn nursery committee received feedback from many physi- cians that they did not recommend the vaccine to the parents because of the low disease incidence in their primarily white patients. This reason for not offering the vaccine at birth was previously mentioned in a survey of medical directors of hospital nurseries. ${ }^{7}$

Because of the continuing challenge of providing universal $\mathrm{HBV}$ vaccination, the standardized nursery orders subsequently changed to enable nurses to administer the vaccine without additional verbal permission from the physician (but with parental consent). Another promising development is the October 2008 endorsement by the National Quality Forum to monitor hospital newborn HBV vaccine coverage. ${ }^{8}$

Figure 2. Change in compliance with American Academy of Pediatrics guidelines for mothers receiving intrapartum antibiotic prophylaxis (IAP) for group B streptococcal (GBS) disease. $* P<.0001 .{ }^{\dagger} P<.005$. CBC, complete blood count.

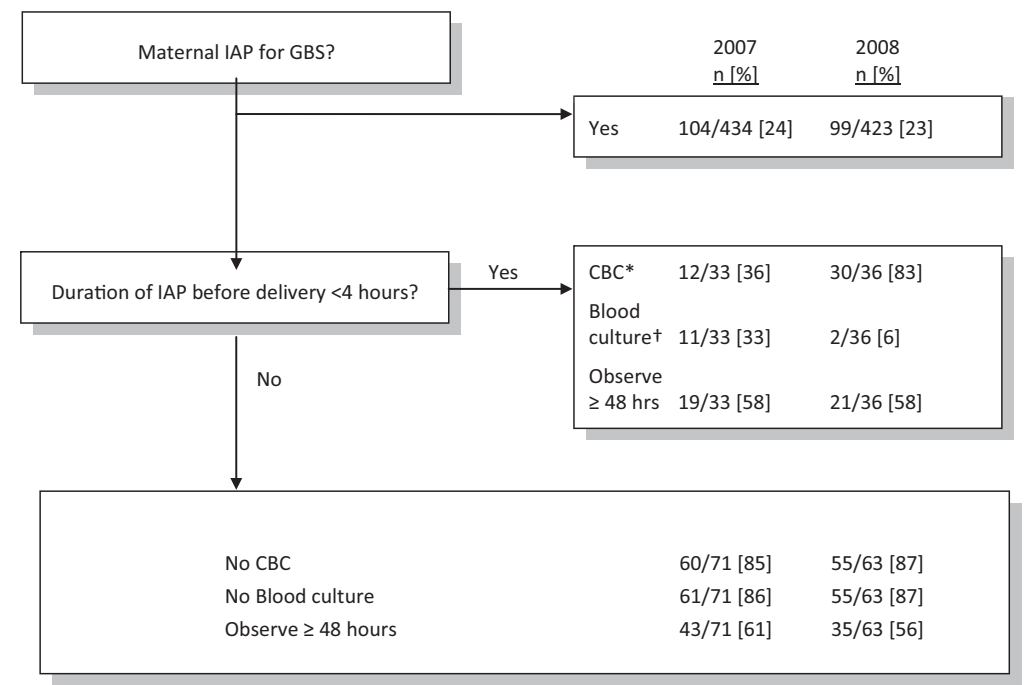


Since 1995, the AAP has recommended a follow-up visit at home or in the clinic within 2 days for newborns who are discharged $<48$ hours after delivery. ${ }^{9}$ In 2004, the AAP expanded their recommendations to include a follow-up visit within 1 to 2 days for infants who are discharged before 72 hours of age. ${ }^{4}$ Many studies have documented that providers do not consistently follow the AAP guidelines for early follow-up of infants discharged $<48$ hours after delivery. ${ }^{10-13}$ This study seems to be the first to report physician compliance with the newer follow-up recommendation for babies discharged before 72 hours of age. A persistent minority of these infants had follow-up recommended a week or more after discharge. The recommendation of the timing of the follow-up visits, not their actual occurrence, was evaluated in this study.

In a 1997 report of 20 pediatricians working in a large community hospital, an educational presentation and letter sent to all pediatricians emphasized the importance of early follow-up of newborns who are discharged early. ${ }^{13}$ This strategy improved the appropriate scheduling of short-stay infants from $22 \%$ to $62 \%$. In addition, the percentage of pediatricians who scheduled early follow-up for all their short-stay infants increased from $0 \%$ to $43 \%$. This hospital newborn nursery committee sent information about the new discharge follow-up recommendations in the standardized orders via E-mail, as well as a subsequent reminder E-mail. The AAP's Safe and Healthy Beginnings project, which includes a toolkit, is designed to facilitate implementation of the 2004 follow-up recommendations. ${ }^{14}$

To our knowledge, compliance with the infant portion of GBS management recommendations has been reported previously in only one study. ${ }^{15}$ In a report from a tertiary medical center in New York, the medical records were reviewed for 537 infants born to mothers with known GBS colonization or with risk factors for it. The recommended evaluation was completed for $29 \%$ of infants. Recommended 48-hour follow-up observation was performed for $65 \%$ of infants with EGA of $\geq 35$ weeks.

The appropriate evaluation rate was better in the current study when compared with this previous report. The change in orders was associated with significant improvement in the rate of $\mathrm{CBC}$ testing. Forty-eight-hour observation rates were lower than reported previously, and they did not improve with the new standardized orders.

It is noteworthy that the aspects of care that improved with the change in standardized orders were those that required little or no physician involvement, specifically HBV immunization and ordering a CBC for infants exposed to IAP for GBS. In contrast, physicians were the sole determinants of LOS and follow-up recommendations, which did not improve despite guidance from the standardized orders. Studies of physician-approved protocols that authorize nurses or other staff members to perform procedures without direct physician involvement (ie, standing orders) have primarily examined their effect on compliance with preventive interventions. ${ }^{16}$ Standing orders have been shown to improve rates of influenza and pneumococcal vaccination, childhood immunizations, and mammogram screening.

This study has some limitations. Because there was no control group, it is possible that other national or state initiatives to improve newborn care could have been responsible for the observed changes, rather than the order sets. Maternal chorioamnionitis is a difficult diagnosis to establish by chart review. The criteria used here may have underestimated the number of cases by excluding 15 women who received clindamycin and vancomycin. Although the newborn nursery standardized orders changed in all the nurseries in the hospital system, the chart review was limited to one hospital. The study hospital provides care for a large number of immigrant patients, primarily from Africa. Therefore, the results may not be applicable to other patient populations.

\section{Conclusion}

The study results demonstrate that a simple change in standardized nursery orders can be associated with significant improvement in newborn care.

\section{References}

1. American Academy of Pediatrics, Committee on Fetus and Newborn. Hospital stay for healthy term newborns. Pediatrics 2010;125:405-9.

2. Schraq S, Gorwitz R, Fultz-Butts K, Schuchat A. Prevention of perinatal group B streptococcal disease. Revised guidelines from CDC. MMWR Recomm Rep 2002;51(RR-11):1-22.

3. Mercier CE, Barry SE, Paul K, et al. Improving newborn preventive services at the birth hospital- 
ization: a collaborative, hospital-based quality-improvement project. Pediatrics 2007;120:481-8.

4. Subcommittee on Hyperbilirubinemia. Management of hyperbilirubinemia in the newborn infant 35 or more weeks of gestation. Pediatrics 2004;114:297-316.

5. Mast EE, Margolis HS, Fiore AE, et al. A comprehensive immunization strategy to eliminate transmission of hepatitis B virus infection in the United States: recommendations of the Advisory Committee on Immunization Practices (ACIP) part 1: immunization of infants, children, and adolescents. MMWR Recomm Rep 2005;54(RR-16):1-31.

6. Willis B, Wortley P, Wang S, Jacques-Carroll L, Zhang F. Gaps in hospital policies and practices to prevent perinatal transmission of hepatitis B virus. Pediatrics 2010;125:704-11.

7. Aiken KD, Clark SJ, Cabana MD. Reasons hospitals give for not offering hepatitis $\mathrm{B}$ vaccine to low-risk newborns. Clin Pediatr 2002;41:681-6.

8. National Quality Forum. National Quality Forum endorses national consensus standards for perinatal care. New NQF-endorsed measures can improve quality of care for mothers and babies. Available at: www.childbirthconnection.org/pdfs/NQF-perinatalmeasures-release.pdf. Accessed 23 January 2011.
9. Hospital stay for healthy term newborns. American Academy of Pediatrics Committee on Fetus and Newborn. Pediatrics 1995;96(4 Pt 1):788-90.

10. Gartner L, Herrarias C, Sebring R. Practice patterns in neonatal hyperbilirubinemia. Pediatrics 1998;101: 25-31.

11. Madlon-Kay DJ. Evaluation and management of newborn jaundice by midwest family physicians. J Fam Pract 1998;47:461-4.

12. Madlon-Kay DJ, Asche SE. Factors that influence the receipt of well baby care in the first 2 weeks of life. J Am Board Fam Med 2006;19:258-64.

13. Maisels M, Kring E. Early discharge from the newborn nursery: effect on scheduling of follow-up visits by pediatricians. Pediatrics 1997;100:72-4.

14. Cash S. Quality improvement innovation network (QuIN). AAP News 2007;28:44.

15. Chandran L, Navaie-Waliser M, Zulqarni NJ, et al. Compliance with group B streptococcal disease prevention guidelines. MCN Am J Matern Child Nurs 2001;26:313-9.

16. Donahue K, Plescia M. Do standing orders help with chronic disease care and health maintenance in ambulatory practice? J Fam Pract 2010;59:226-7. 